\title{
SALARIOS Y PAGOS AL CAPITAL EN LA INDUSTRIA MEXICANA
}

\author{
Adalberto García Rocha \\ El Colegio de México
}

\section{INTRODUCCIÓN}

EN ESTE TRABajo se examina la distribución del ingreso entre trabajo y capital en la industria mexicana, en el periodo 1965-1970. La investigación es de carácter exploratorio y se basa en información censal. Se estudian las relaciones entre tamaño de empresa, productividad, ganancias, salarios, y la participación de estos últimos en el valor agregado industrial. Aunque la mecánica simple de las estructuras de costos de las empresas es insuficiente para estudiar la formación del salario y la tasa de ganancias, se extraen de las cifras algunas inferencias sobre los orígenes de la desigualdad del ingreso. No obstante la naturaleza empírica y descriptiva del trabajo presente, se ha creído necesario hacer algunas aclaraciones previas sobre cuánto puede el libro de texto ayudar a la interpretación de datos en cuestiones distributivas. Esto se hace en el resto de la presente introducción y en la sección II del documento, y en las partes III y IV se examina la evidencia empírica de los censos industriales.

Aunque todas las escuelas actuales de teoría económica provienen del análisis clásico de la distribución del ingreso entre clases sociales, la literatura moderna muestra una clara división de orientaciones. Para una de las corrientes - la teoría de la ofelimidad o neoclásica- 1a distribución del ingreso es tema subsidiario del análisis de la asignación de recursos en la producción y el consumo. En esta teoría el salario y la tasa de ganancias surgen de las preferencias de los consumidores condicionadas por la física de la producción. Para otras corrientes, como la marxista o la neokeynesiana, la distribución del ingreso es un fenómeno en esencia político y punto de partida para el análisis. El salario y la tasa de ganancia son para estas últimas escuelas el resultado de conflictos de poder entre grupos de interés.

La divergencia de orientación concierne a la naturaleza y objetivos de la ciencia económica y pone a ésta en una situación incierta. No obstante, la escuela de la utilidad predomina en la enseñanza occidental, es practicada por los más eminentes economistas, y da para hacer reco- 
mendaciones de política económica. Las otras corrientes subsisten en los medios académicos no tanto como enfoques alternativos, sino como especializaciones de un extraño tronco común de teoría.

La teoría de la ofelimidad reclama un lugar entre las ciencias positivas, como la termodinámica, donde la validez de las hipótesis depende estrictamente de su coincidencia con hechos observados. En sus versiones más avanzadas, dicha teoría es una construcción axiomática donde las hipótesis a verificar son inferidas por deducción de un conjunto de postulados. Sobre esto último es pertinente señalar que el método deductivo no es el único medio de construir hipótesis o teoremas verificables. La física teórica moderna, por ejemplo, fue motivada por la necesidad de unificar hipótesis conjeturadas y manipuladas experimentalmente mucho antes de la existencia de un sistema formal. ${ }^{1}$

En la teoría de la ofelimidad se observa un afán por proceder en sentido inverso al anterior. Casi todo el esfuerzo se ha puesto en construir un aparato axiomático altamente especulativo y con una base empírica fragmentaria. Es así que la enseñanza de la economía se ha cubierto prematuramente de una mística deductiva y las matemáticas se han vuelto lengua litúrgica del economista moderno. Tal fundamentalismo intelectual ha creado en el estudiante y no pocos profesionales la impresión de que el método deductivo tiene valor empírico intrínseco y de que ninguna hipótesis puede ser interesante si no proviene de un elaborado razonamiento simbólico.

Por otro lado, la pretensión positivista suele partir de una interpretación superficial de la teoría del conocimiento. En realidad el método positivo genera demandas demasiado fuertes sobre una ciencia incipiente, con la consecuencia de que la gran mayoría de los autores encuentran imposible apegarse al rigor de dicho método. El lector descubrirá hasta en las argumentaciones más depuradas, términos y razonamientos en abierta violación al canon positivista. Verá que conceptos tales como eficiencia, racionalidad, estados óptimos, mercados deformes, y otros, son evocados sin mayor aclaración de la diferencia entre sus significados coloquial y técnico.

Estas consideraciones tienen importancia particular en el análisis de custiones distributivas dada la dificultad tan frecuente de distinguir entre el afán de los autores por explicar los fenómenos y el de modificarlos. Tal suele ser el caso cuando se compara una economía capitalista y una economía competitiva hipotética, como la del libro de texto, cuyo único propósito es definir estados de equilibrio sin mayor pretensión causal. ${ }^{2}$

La característica más importante del discurso deductivo es que la interpretación de los términos no es única. Sin embargo, se insiste en

1 Vale recordar que la fundamentación formal de las matemáticas mismas es muy reciente.

2 La postura más clara a este respecto puede verse en F. Hahn, On the Notion of Equilibrium in Economics, Cambridge University Press, 1973, p. 32. 
dar a los modelos de equilibrio general o parcial interpretaciones en una sola dirección. Conviene añadir que algunas proposiciones que se derivan únicamente de la formulación del problema a menudo se atribuyen a una teoría en particular. ${ }^{3}$ Sobre estos puntos se hablará en la sección siguiente.

\section{Clases sociales y factores de producción}

La teoría del valor trabajo fue desterrada de la ciencia económica occidental por sus connotaciones políticas, en especial en asuntos obreropatronales. Para sustituirla se creó el concepto de utilidad $\mathfrak{u}$ ofelimidad cardinal como medida del valor de un bien. Sin embargo, este concepto fue de nuevo esgrimido por liberales y socialistas para argumentar en favor de la igualdad de ingresos. Hubo entonces que idear la noción de utilidad ordinal, ajena a comparaciones interpersonales de felicidad, que dio lugar a lo que hoy se conoce como espacio de preferencia. Se añadieron luego una teoría de la producción y supuestos sobre los mercados, y se llegó así a la teoría moderna de los precios, entre los cuales se encuentran el salario y la tasa de ganancia.

La teoría de la producción neoclásica trata de la existencia de alternativas técnicas mediante las cuales los consumidores pueden equilibrar sus deseos de bienes con la aversión al trabajo. Aunque el suministro de trabajo y capital proviene de los individuos, la oferta de factores de producción es independiente de la estructura de derechos de propiedad. Los supuestos de competencia perfecta en todos los mercados eliminan el efecto de coaliciones o cualquier influencia política sobre los precios. A diferencia de las teorías de los clásicos, en la neoclásica ningún factor de producción tiene un precio residual. Todos los factores son escasos. El precio de cada uno es a la vez un costo de producción y resulta de una ley física, por lo común expresada como la igualdad entre el salario y la tasa de ganancia con las productividades marginales del trabajo y el capital, respectivamente. En el caso del trabajo no existen una jornada o el empleo como fenómeno contractual entre obreros y patrones. En la economía competitiva todo mundo puede trabajar cuanto quiera sin régimen ocupacional alguno. Existe pleno empleo. Todo individuo puede también ser propietario y obtener ingresos por capital, según dicten sus preferencias por el ahorro. No hay capitalistas, obreros ni rentistas en la acepción usual, porque los individuos tienen abierta la posibilidad de participar en la producción en la forma que gusten. Esta situación tiene cierta semejanza con el llamado capitalismo popular, donde todos los individuos son propietarios de los bienes de producción en forma descentralizada. ${ }^{4}$

3 En B. Rusell, The Problem of Philosophy, Oxford University Press, 1973, se discute en detalle la naturaleza del método positivo en la ciencia moderna.

4 Buena parte de los conceptos de esta descripción y los de las secciones II 
Dentro del contexto utilitario resulta entonces inexacto hablar de distribución del ingreso entre individuos, ya que la distribución de la propiedad no interviene en el análisis. Este es precisamente el capítulo que falta a la teoría de la ofelimidad..$^{5}$ Puede sólo examinarse la distribución de los pagos al trabajo y al capital como factores disociados de individuos, y es así como deben interpretarse los teoremas neoclásicos.

Son frecuentes en la literatura las afirmaciones en el sentido de que los precios no coinciden con sus productividades o utilidades marginales debido a imperfecciones en los mercados. Esta forma de razonar no sólo es circular sino indebida desde el punto de vista del método positivo. La teoría sirve para explicar fenómenos y no para censurar a los hechos por no apegarse a ella. Si los precios no corresponden con el modelo, es éste el que hay que cambiar. Por otra parte, como la deducción involucra varias premisas, no es posible establecer cuál de ellas debe modificarse. El razonamiento es circular porque si las predicciones deducidas no coinciden con lo observado es evidente que hay que cambiar los supuestos, es decir, hacer otra teoría.

La intención de hacer mercados perfectos pertenece al terreno de lo valorativo. Para fijar las ideas, un mercado perfecto no es peor o mejor que uno imperfecto $o$ un consumidor racional mejor que uno irracional. Para ser objetivos todos estos términos deben desvestirse de cualquier connotación que no sea estrictamente clasificatoria. La teoría neoclásica no explica cómo un mercado transcurre de lo perfecto a lor imperfecto o viceversa. El tipo de mercado es un artificio para clasificar situaciones, pero no en un sentido histórico o causal.

Desde el ángulo ingenieril, las contribuciones del trabajo o el capital al producto pueden definirse con precisión razonable, y lo mismo puede decirse del proceso físico de sustitución entre trabajo y capital. Sin embargo, al hablar de pagos a los factores de producción en términos más generales el concepto de contribución al producto se torna ambiguo o inútil. Por ejemplo, existen grupos de individuos que perciben ingresos y no contribuyen físicamente a producirlo (herederos, militares, espe-

y III han sido tomados de C. J. Bliss, Capital Theory and the Distribution of Income, North-Holland, 1975, en particular la parte IV.

5 Existen en la muy abundante literatura sobre distribución varios esfuerzos por incorporar al análisis de la utilidad una teoría de la distribución entre individuos. En J. Pen, Income Distribution, Praeger, 1976, el lector encontrará una descripción general del análisis empírico de la distribución del ingreso. J. E. Stiglitz, "Distribution of Income and Wealth among Individuals", en A. B. Atkinson (Comp.), Wealth, Income and Inequality presenta una discusión más específica sobre la distribución en el modelo neoclásico. En el conocido trabajo de S. Kuznetz, "Economic Growth and Income Inequality", The American Economic Review, marzo de 1955 , pp. 24-28, se encuentran predicciones sobre la conducta probable de la distribución del ingreso en paises subdesarrollados. Este último estudio es de los primeros de la corriente que sostiene que la desigualdad del ingreso aumenta en las primeras etapas del desarrollo pero eventualmente disminuye. 
culadores, amas de casa y niños ricos, rentistas, ancianos pensionados, etc.). Por otra parte, en ningún país del mundo la población activa excede de la mitad de la población total. Por lo tanto, para explicar la distribución del ingreso entre activos e inactivos es ineludible acudir a fenómenos institucionales como la familia, los sistemas de seguridad social, organismos de defensa y los derechos de propiedad.

Las leyes físicas de producción podrían tener relación con la distribución del ingreso entre los individuos activos. Es por esta razón que la distribución del ingreso de la teoría económica suele referirse en exclusiva a situaciones de empleo pleno, es decir, al fenómeno de distribución entre aquellos individuos vinculados con la producción. De alguna manera se busca verificar la hipótesis de que las remuneraciones corresponden a cierto esfuerzo o contribución al producto. Es aquí donde surge la ambigüedad. No es lo mismo la función de una máquina en el proceso de producción que la parte del producto que va a dar al propietario de la máquina. En la función de producción neoclásica no hay capitalistas sino máquinas. En casos como el de los rentistas el concepto de contribución al producto resulta todavía más ambiguo. Los ingresos del rentista o el capitalista son algo completamente distinto de la productividad marginal del capital como factor de producción.

Por estas razones, la inspección de los datos y las conjeturas que se discuten en las secciones siguientes no parten de hipótesis formales del libro de texto. Se ha preferido examinar las cifras con el propósito exclusivo de ver qué muestran acerca de la distribución funcional del ingreso y sus tendencias en las empresas manufactureras. ${ }^{6}$ De este modo se evitan los conocidos ejercicios econométricos para hacer que las cifras se ajusten a especificaciones preconcebidas.

\section{Productividad, Salarios y ganancias}

En los cuadros 1 y 2 aparecen algunas características de la producción manufacturera mexicana en los años 1965 y 1970, según la información de los censos industriales. Las empresas aparecen clasificadas en los cuadros en nueve categorías de tamaño en términos del número de obreros.

Lo más notable de las cifras son las diferencias de productividad, salarios y activos. Este fenómeno es reflejo de los elevados niveles de concentración industrial en México: en 1970, $2.1 \%$ del total de empre-

6 S. Trejo, Industrialización y empleo en México, México, Fondo de Cultura Económica, 1973, elabora un análisis del crecimiento industrial de México en líneas semejantes a las del artículo presente. Existen varias descripciones sobre el crecimiento industrial en México, aunque en general pocas dan atención a la distribución del ingreso: véase por ejemplo: NAFINSA-CEPAL, La politica industrial en el desarrollo económico de México, México, 1977. 
Cuadro 1

México: Características de la industria, 1965 Y 1970

\begin{tabular}{|c|c|c|c|c|c|}
\hline Tamañoa & $\begin{array}{c}\text { Número de } \\
\text { estableci- } \\
\text { mientos }\end{array}$ & $\begin{array}{l}\text { Numero de } \\
\text { trabajadores } \\
(L)\end{array}$ & $\begin{array}{c}\text { Nómina } \\
\text { total } \mathrm{b} \\
(W)\end{array}$ & $\begin{array}{l}\text { Valor de } \\
\text { activos }^{\mathrm{b}}\end{array}$ & $\begin{array}{c}\text { Valor } \\
\text { agregado b } \\
(V)\end{array}$ \\
\hline \multicolumn{6}{|c|}{1965} \\
\hline Menos de 5 & $\begin{array}{r}41838 \\
(66.7)\end{array}$ & $\begin{array}{c}115530 \\
(9.4)\end{array}$ & $\begin{array}{c}480.853 \\
(2.6)\end{array}$ & 1227193 & $\begin{array}{c}1411421 \\
(3.3)\end{array}$ \\
\hline $6-15$ & $\begin{array}{r}10275 \\
(16.4)\end{array}$ & $\begin{array}{c}92547 \\
(7.5)\end{array}$ & $\begin{array}{c}757960 \\
(4.2)\end{array}$ & $\begin{array}{c}2977124 \\
(3.2)\end{array}$ & $\begin{array}{c}1859869 \\
(4.4)\end{array}$ \\
\hline $16-25$ & $\begin{array}{r}3144 \\
(5.0)\end{array}$ & $\begin{array}{c}62131 \\
(5.1)\end{array}$ & $\begin{array}{c}681617 \\
(3.9)\end{array}$ & $\begin{array}{c}3009859 \\
(3.3)\end{array}$ & $\begin{array}{c}1606307 \\
(3.8)\end{array}$ \\
\hline $26-50$ & $\begin{array}{l}3101 \\
(4.9)\end{array}$ & $\begin{array}{c}111614 \\
(9.1)\end{array}$ & $\begin{array}{c}1378975 \\
(7.6)\end{array}$ & $\begin{array}{c}6600446 \\
(7.2)\end{array}$ & $\begin{array}{c}3226839 \\
(7.6)\end{array}$ \\
\hline $51-75$ & $\begin{array}{l}1312 \\
(2.1)\end{array}$ & $\begin{array}{c}80882 \\
(6.6)\end{array}$ & $\begin{array}{c}1110144 \\
(6.2)\end{array}$ & $\begin{array}{c}5196217 \\
(5.7)\end{array}$ & $\begin{array}{c}2449787 \\
(5.7)\end{array}$ \\
\hline $76-100$ & $\begin{array}{r}750 \\
(1.2)\end{array}$ & $\begin{array}{c}63788 \\
(5.2)\end{array}$ & $\begin{array}{c}915752 \\
(5.1)\end{array}$ & $\begin{array}{c}4329213 \\
(4.7)\end{array}$ & $\begin{array}{c}2098984 \\
(4.9)\end{array}$ \\
\hline $101-250$ & $\begin{array}{r}1407 \\
\cdot(2.2)\end{array}$ & $\begin{array}{c}223628 \\
(18.2)\end{array}$ & $\begin{array}{c}3569459 \\
(19.8)\end{array}$ & $\begin{array}{c}18325918 \\
(20.0)\end{array}$ & $\begin{array}{c}8302198 \\
(19.5)\end{array}$ \\
\hline $251-500$ & $\begin{array}{r}621 \\
(1.0)\end{array}$ & $\begin{array}{c}200691 \\
(16.3)\end{array}$ & $\begin{array}{c}3501341 \\
(19.4)\end{array}$ & $\begin{array}{c}18114381 \\
(19.8)\end{array}$ & $\begin{array}{c}8164495 \\
(19.1)\end{array}$ \\
\hline Más de 500 & $\begin{array}{l}306 \\
(.5)\end{array}$ & $\begin{array}{c}277415 \\
(22.6)\end{array}$ & $\begin{array}{c}5645635 \\
(31.3)\end{array}$ & $\begin{array}{c}31926088 \\
(34.8)\end{array}$ & $\begin{array}{c}13523.342 \\
(31.7)\end{array}$ \\
\hline $\begin{array}{l}\text { Total de } \\
\text { empresas }\end{array}$ & 62754 & 1228226 & 18041736 & 91706439 & 42642.242 \\
\hline \multicolumn{6}{|c|}{1970} \\
\hline Menos de 5 & $\begin{array}{c}38953 \\
(63.3)\end{array}$ & $\begin{array}{c}106512 \\
(7.5)\end{array}$ & $\begin{array}{c}712659 \\
(2.2)\end{array}$ & $\begin{array}{c}2163922 \\
(1.4)\end{array}$ & $\begin{array}{c}2062404 \\
(2: 6)\end{array}$ \\
\hline $6-15$ & $\begin{array}{l}10711 \\
(17.4)\end{array}$ & $\begin{array}{c}96502 \\
(6.8)\end{array}$ & $\begin{array}{c}1139974 \\
(3.6)\end{array}$ & $\begin{array}{c}4485911 \\
(3.0)\end{array}$ & $\begin{array}{c}2794705 \\
(3.6)\end{array}$ \\
\hline $16-25$ & $\begin{array}{r}3251 \\
(5.3)\end{array}$ & $\begin{array}{c}65529 \\
(4.6)\end{array}$ & $\begin{array}{c}1022506 \\
(3.2)\end{array}$ & $\begin{array}{c}4373952 \\
(2.9)\end{array}$ & $\begin{array}{c}2560472 \\
(3.3)\end{array}$ \\
\hline $26-50$ & $\begin{array}{r}3372 \\
(5.5)\end{array}$ & $\begin{array}{c}121452 \\
(8.5)\end{array}$ & $\begin{array}{c}2092669 \\
(6.6)\end{array}$ & $\begin{array}{c}9391405 \\
(6.2)\end{array}$ & $\begin{array}{c}4929156 \\
(6.3)\end{array}$ \\
\hline $51-75$ & $\begin{array}{r}1508 \\
(2.4)\end{array}$ & $\begin{array}{c}94405 \\
(6.6)\end{array}$ & $\begin{array}{c}1842217 \\
(5.8)\end{array}$ & $\begin{array}{c}8656543 \\
(5.8)\end{array}$ & $\begin{array}{c}4372913 \\
(5.6)\end{array}$ \\
\hline $76-100$ & $\begin{array}{r}850 \\
(1.3)\end{array}$ & $\begin{array}{c}76120 \\
(5.3)\end{array}$ & $\begin{array}{c}1616224 \\
(5.1)\end{array}$ & $\begin{array}{c}7446708 \\
(5.0)\end{array}$ & $\begin{array}{c}3797621 \\
(4.9)\end{array}$ \\
\hline $101-250$ & $\begin{array}{l}1721 \\
(2.8)\end{array}$ & $\begin{array}{c}269874 \\
(18.9)\end{array}$ & $\begin{array}{c}6193399 \\
(19.5)\end{array}$ & $\begin{array}{c}29758566 \\
(19.8)\end{array}$ & $\begin{array}{c}15607430 \\
(19.9)\end{array}$ \\
\hline $251-500$ & $\begin{array}{r}670 \\
(1.1)\end{array}$ & $\begin{array}{c}229142 \\
(16.0)\end{array}$ & $\begin{array}{c}5926485 \\
(18.6)\end{array}$ & $\begin{array}{c}29915428 \\
(19.9)\end{array}$ & $\begin{array}{c}14716920 \\
(18.8)\end{array}$ \\
\hline Más de 500 & $\begin{array}{r}443 \\
(1.0)\end{array}$ & $\begin{array}{c}368830 \\
(25.8)\end{array}$ & $\begin{array}{c}11214845 \\
(35.3)\end{array}$ & $\begin{array}{c}54145436 \\
(36.0)\end{array}$ & $\begin{array}{c}27562.225 \\
(35.1)\end{array}$ \\
\hline $\begin{array}{l}\text { Total de } \\
\text { empresas }\end{array}$ & 61479 & 1428366 & 31760978 & 150337871 & 78443846 \\
\hline
\end{tabular}

Fuente: Censos Industriales de 1965 y 1970, México, Dirección General de Estadística. Las cifrasi se obtuvieron por agregación e interpolación de los cuadros, a nivel de cuatro dígitos de la clasificación censal.

- Tamaño de los establecimientos según el número de trabajadores.

- Miles de pesos a precios corrientes. 
sas produjeron $50.8 \%$ del valor agregado. Para apreciar mejor este punto se ha construido la gráfica 1 que contiene para 1965 curvas de Lorenz de valor agregado, salario medio y activos como abscisas y el número de obreros como ordenada. Las cifras correspondientes a 1970 dan curvas de Lorenz prácticamente iguales a las de 1965 y por ello no se han trazado en la gráfica. Estas muestran diferencias de productividad (valor

\section{Cuadro 2}

MÉxico: CARACTERÍSTiCAS DE LA INDUSTRIA, 1965 Y 1970

\begin{tabular}{|c|c|c|c|c|}
\hline Tamaño & $\begin{array}{c}\text { Salario } \\
\text { medio } \\
\text { anual }\end{array}$ & $\begin{array}{l}\text { Valor de los } \\
\text { activos por } \\
\text { trabajador } \mathrm{b}\end{array}$ & $\begin{array}{l}\text { Valor agregado } \\
\text { por trabajador }^{\mathrm{b}}\end{array}$ & $\begin{array}{l}\text { Valor agregado } \\
\text { menos nómina } \\
\text { global } \mathrm{c}\end{array}$ \\
\hline \multicolumn{5}{|c|}{1965} \\
\hline $\begin{array}{c}\text { Menos de } 5 \\
6-15 \\
16-25 \\
26-50 \\
51-75 \\
76-100 \\
101-250 \\
251-500 \\
\text { Más de } 500 \\
\text { Total de empresas }\end{array}$ & $\begin{array}{rl}4 & 162 \\
8 & 190 \\
10 & 971 \\
12 & 355 \\
13 & 725 \\
14356 \\
15962 \\
17446 \\
20351 \\
14689\end{array}$ & $\begin{array}{r}10622 \\
32169 \\
48444 \\
59136 \\
64244 \\
67869 \\
81948 \\
90260 \\
115084 \\
74666\end{array}$ & $\begin{array}{l}12217 \\
20096 \\
25854 \\
28911 \\
30288 \\
32906 \\
37125 \\
40682 \\
48748 \\
34719\end{array}$ & $\begin{array}{r}930568 \\
1101909 \\
924690 \\
1847864 \\
1339643 \\
1183232 \\
4732739 \\
4663154 \\
7877707 \\
24600506\end{array}$ \\
\hline \multicolumn{5}{|c|}{1970} \\
\hline $\begin{array}{l}\text { Menos de } 5 \\
6-15 \\
16-25 \\
26-50 \\
51-75 \\
76-100 \\
101-250 \\
251-500 \\
\text { Más de } 500 \\
\text { Total de empresas }\end{array}$ & $\begin{array}{r}6691 \\
11813 \\
15604 \\
17230 \\
19514 \\
21233 \\
22949 \\
125864 \\
30407 \\
22236\end{array}$ & $\begin{array}{r}20316 \\
46485 \\
66748 \\
77326 \\
91696 \\
97828 \\
110268 \\
130554 \\
146803 \\
105252\end{array}$ & $\begin{array}{l}19363 \\
28960 \\
39074 \\
40585 \\
46321 \\
49890 \\
57832 \\
64226 \\
74729 \\
54919\end{array}$ & $\begin{array}{r}1349745 \\
1654731 \\
1537966 \\
2836487 \\
2530696 \\
2181397 \\
9414031 \\
8790435 \\
16347380 \\
46682868\end{array}$ \\
\hline
\end{tabular}

a Tamaño de los establecimientos según el número de trabajadores.

b Pesos corrientes.

c Miles de pesos.

agregado por obrero) muty semejantes a las de salarios, y una mayor concentración de los activos. La estructura de disparidades sugiere cierta ineficiencia en la producción, ya que las empresas elevan las diferencias de productividad a base de una mayor concentración de los activos, es decir, de mayores gastos de inversión por obrero.

El cuadro 3 contiene índices de concentración y las participaciones del salario en el valor agregado por rama industrial. Los índices corro- 


\section{Gráfica 1}

Productividad Media, SALARIOS Y activos poR TRABA JAdOR

(Los números se refieren a tamaños)



Cuadro 3

MÉXICO: INDICES DE CONCENTRACIÓN Y PARTICIPACIÓN DEL SALARIO EN EL VALOR AGREGADO, POR RAMA INDUSTRIAL, 1965 Y 1970

\begin{tabular}{|c|c|c|c|c|c|c|}
\hline \multirow{2}{*}{\multicolumn{2}{|c|}{ Rama }} & \multicolumn{2}{|c|}{ Coeficiente de Gini } & \multicolumn{3}{|c|}{ Participaciones } \\
\hline & & \multirow{2}{*}{$\frac{1965}{0.709}$} & \multirow{2}{*}{$\frac{1970}{0.720}$} & \multirow{2}{*}{$\frac{1965}{0.41}$} & \multirow{2}{*}{$\frac{1970}{0.35}$} & \multirow{2}{*}{$\frac{\text { Cambio (\%) }}{-14.60}$} \\
\hline 20 & Alimentos & & & & & \\
\hline 21 & Bebidas & 0.812 & 0.822 & 0.39 & 0.34 & -12.57 \\
\hline 22 & Tabaco & 0.772 & 0.663 & a & a & \\
\hline 23 & Textil & 0.686 & 0.692 & 0.49 & 0.50 & 0.15 \\
\hline 24 & Calzado y Ropa & 0.694 & 0.719 & 0.50 & 0.49 & -1.51 \\
\hline 25 & Madera y Corcho & 0.800 & 0.758 & 0.46 & 0.47 & 1.11 \\
\hline 26 & Muebles & 0.669 & 0.655 & 0.56 & 0.52 & -6.72 \\
\hline 27 & Papel & 0.710 & 0.705 & 0.41 & 0.42 & 1.71 \\
\hline 28 & Imprenta & 0.699 & 0.705 & 0.51 & 0.51 & 0.00 \\
\hline 29 & Cuero & 0.647 & 0.641 & 0.50 & 0.44 & -11.62 \\
\hline 30 & Hule & 0.771 & 0.768 & 0.42 & 0.34 & -17.33 \\
\hline 31 & Química & 0.709 & 0.713 & 0.39 & 0.38 & -1.91 \\
\hline 32 & Derivados de Petróleo & 0.683 & 0.708 & 0.32 & 0.25 & -21.96 \\
\hline 33 & No metálicos & 0.758 & 0.763 & 0.43 & 0.45 & 4.33 \\
\hline 34 & Metálica básica & 0.666 & 0.727 & 0.34 & 0.35 & 3.39 \\
\hline 35 & Productos Metálicos & 0.774 & 0.774 & 0.50 & 0.47 & -6.02 \\
\hline 36 & Máquina no eléctrica & 0.734 & 0.737 & 0.49 & 0.44 & -10.38 \\
\hline \multirow[t]{2}{*}{37} & Electricidad y & & & & & \\
\hline & Electrónica & 0.728 & 0.705 & 0.41 & 0.44 & 7.35 \\
\hline 38 & Automotriz & 0.708 & 0.802 & 0.45 & 0.42 & -7.74 \\
\hline 39 & Otros & $0.661^{l}$ & 0.714 & 0.43 & 0.48 & 12.40 \\
\hline
\end{tabular}

a El sector 22 (Tabaco), fue eliminado de los cálculos por fallas en las cifras. El coeficiente de Gini mide las desigualdades de tamaños: es cero cuando las desigualdades son nulas y $1 / 19$ cuando la concentración es máxima. 
boran lo dicho antes, acerca de la elevada concentración de los merca. dos, y no requieren mayor comentario.

En la gráfica 2 se han trazado los niveles de productividad y salarios en cada uno de los nueve tamaños contra el valor de los activos por obrero, para 1965 y 1970. En ambos años se observa que las relaciones son prácticamente lineales, y que la productividad (las dos líneas superiores) y los salarios (las líneas inferiores) aumentan más lentamente que el capital por obrero, es decir, en todos los casos las pendientes de las curvas son menores que la unidad. Entre los dos años se observa también un desplazamiento hacia arriba, debido en parte a cambios en los precios del producto y los bienes de capital.

Los salarios aumentan más lentamente que la productividad ante cambios en el capital por obrero tanto en corte transversal - a lo largo de los tamaños- como en el tiempo. Entre un año y otro puede verse, por ejemplo, que si se traza una línea entre los puntos con el número 4 en las líneas de productividad su pendiente sería mayor a la correspondiente a las líneas de salarios. En corte transversal se aprecia en la gráfica que las líneas de productividad tienen pendiente mayor que las de salarios.

En las columnas 4,5 y 6 del cuadro 4 se aprecia que entre 1965 y 1970 la productividad creció proporcionalmente más rápido que los activos por obrero en todos los tamaños salvo los dos menores (menos de 15 obreros). Las empresas grandes elevan su ingreso o valor agregado por obrero proporcionalmente más rápido que las pequeñas en relación a los activos por obrero (véase la columna 6 del cuadro 4 ).

Juntas, las consideraciones anteriores indican que las relaciones de productividad no coinciden con los supuestos de curvatura del modelo neoclásico de producción, o al menos hacen muy improbables a dichos supuestos. Por una parte, la relación entre productividad y activos en el sentido de los tamaños es lineal y no muestra márgenes decrecientes (concavidad). Al aumentar el capital por obrero no se observan rendimientos marginales decrecientes.

Puede suponerse por otra parte que cada categoría de tamaños tiene leyes de producción propias, como sugieren las curvas $F_{1}$ y $F_{3}$. Aunque la curvatura no puede juzgarse con sólo dos observaciones en el tiempo, puede verse que las funciones de producción quedan anidadas en forma tal que las empresas mayores tienen tecnologías inferiores a las pequeñas. Las empresas de tamaño 1 podrían producir más que las de tamaño 3 con una misma dotación de capital por obrero. La disposición de los puntos en la gráfica es tal que las empresas aumentan de tamaño pero en la dirección de cambio técnico o eficiencia menor. Resulta entonces que el nivel de producción global sería mucho mayor si las empresas tuviesen todas la tecnología de las de tamaño menor.

Podría argumentarse que las gráficas no permiten inferencias como las anteriores por estar construidas con valores monetarios y no con 
Cuadro 4

INDICADORES DE CAMBIO EN LA COMPOSICIÓN DEL VALOR AGREGADO

\begin{tabular}{|c|c|c|c|c|c|c|c|c|c|c|}
\hline \multirow{2}{*}{ Tamaño } & \multicolumn{2}{|c|}{$W / L$} & \multicolumn{4}{|c|}{ Cambios relativos } & \multicolumn{2}{|c|}{$\begin{array}{c}\text { Ganancias } \\
\div \text { valor de activos }\end{array}$} & \multicolumn{2}{|c|}{$\begin{array}{l}\text { Ganancias por empresa } \\
\div \text { ganancias de la menor }\end{array}$} \\
\hline & 1965 & 1970 & $W / V$ & $V / L$ & $K / L$ & $\left(\frac{V}{L} / \frac{K}{L}\right)$ & 1965 & 1970 & 1965 & 1970 \\
\hline Menos de 5 & 0.341 & 0.346 & 46.60 & 58.49 & 91.26 & 0.64 & 0.7583 & 0.6237 & 1.0 & 1.0 \\
\hline $5-15$ & 0.408 & 0.408 & 0.00 & 44.11 & 44.50 & 0.99 & 0.3701 & 0.3689 & 4.8 & 4.5 \\
\hline $16-25$ & 0.424 & 0.399 & -5.90 & 51.13 & 37.78 & 1.35 & 0.3072 & 0.3516 & 13.2 & 13.6 \\
\hline $26-50$ & 0.427 & 0.425 & -0.47 & 40.38 & 30.76 & 1.31 & 0.2800 & 0.3020 & 26.8 & 24.3 \\
\hline 51.75 & 0.453 & 0.421 & -7.07 & 52.94 & 42.73 & 1.24 & 0.2578 & 0.2933 & 45.9 & 48.9 \\
\hline $76-100$ & 0.436 & 0.426 & -2.29 & 51.61 & 44.14 & 1.17 & 0.2733 & 0.2929 & 70.9 & 74.1 \\
\hline $101-250$ & 0.430 & 0.397 & -7.67 & 55.78 & 34.56 & 1.61 & 0.2583 & 0.3163 & 151.2 & 157.9 \\
\hline $251-500$ & 0.429 & 0.403 & -6.06 & 57.87 & 44.64 & 1.30 & 0.2574 & 0.2938 & 337.6 & 378.6 \\
\hline Más de 501 & 0.417 & 0.407 & -2.40 & 53.30 & 27.56 & 1.93 & 0.2467 & 0.3019 & 1157.5 & 1064.9 \\
\hline $\begin{array}{l}\text { Total de } \\
\text { empresas }\end{array}$ & 0.423 & 0.405 & -4.26 & 58.18 & 40.96 & 1.42 & 0.2683 & 0.3105 & & \\
\hline
\end{tabular}




\section{Gráfica 2}

CuRvas de LoRenz de valor agregado (v), SAlarios (s) y valor DE ACtivos (A). MÉXICO, 1965




índices físicos de capital y producto. Sin embargo, esta objeción no afecta las conclusiones porque la función de producción tiene la misma curvatura en términos monetarios que en términos físicos si los precios son constantes. ${ }^{7}$ Por último, podría suponerse que cada punto observado pertenece a una función de producción diferente. En tal caso, de cualquier manera se preservaría la posición relativa de las empresas grandes y pequeñas en el sentido de los tamaños. Sin embargo, las gráficas no contienen información suficiente para hacer mayores inferencias. ${ }^{8}$

Ante tales diferencias de tamaños, productividad y salarios, resulta inadecuado el supuesto de que las empresas se enfrentan a precios iguales determinados por la física de producción. Existen sin duda relaciones monopólicas y monopsónicas en las transacciones. Esto es más probable aun si se considera que las cifras se refieren a todo el pais y por lo tanto ocultan diferencias regionales. Es así que la interpretación de la productividad monetaria o nominal en términos de su contraparte física se torna confusa. Las empresas grandes tienen mayor valor agregado por obrero por muchas causas y no particularmente porque sean las más productivas en el sentido físico o de organización. Es por lo tanto preferible interpretar las diferencias de productividad nominal como diferencias de ingreso. Dicho de otro modo, el hecho de que los obreros de las empresas más grandes generen cinco veces más valor por obrero que las pequeñas indica simplemente que el total del valor agregado resulta distribuido en esa forma. Las razones pueden ser de índole muy diversa: rentas monopólicas en el mercado de productos, economías de escala, mayor volumen físico de producción de las empresas grandes, diferencias en el impacto de exenciones, subsidios y protección de mercados, mayor calificación de la fuerza de trabajo de las empresas grandes, etc. Para los fines del trabajo presente no es necesario explorar las causas sino las consecuencias de las diferencias en el valor agregado por obrero. Las "diferencias de productividad" y eficiencia no pueden atribuirse directamente a diferencias en destreza productiva física sin un análisis paralelo de la estructura de precios.

7 Con rendimientos constantes a escala, la relación $Y=f(K, L)$ entre ingreso $(Y)$, capital $(K)$ y trabajo $(L)$ puede escribirse $y=g(k)$, donde $y=K / L$ es la productividad física media del trabajo y $k=K / L$ la "cantidad" de capital por obrero. Si $p_{k}$ es el precio del capital y $h\left(p_{k} k\right)$ el valor de la productividad a precios corrientes, entonces $(d g / d k)=(d g / d h)(d h / d g)=h^{\prime}\left(p_{k} k\right)$. Por lo tanto $h^{\prime}\left(p_{k} k\right)=$ $\left(1 / p_{k}\right)(d g / d k)$ tiene curvatura del mismo signo que $g$ si $p_{k}$ es constante. Las variables monetarias deben entonces mostrar rendimientos marginales decrecientes si las magnitudes físicas cumplen dicha ley.

8 En el trabajo de S. Trejo, Industrialización y empleo, se estiman funciones de producción (del tipo Cobb-Douglas) con información de 1965 dentro de cada grupo de tamaños. No interesa aquí discutir los resultados del estudio anterior a la luz de los del trabajo presente, sino señalar que en el estudio citado se afirma que "existen varias dudas sobre la hipótesis de que las empresas de mayor ta. maño son las más eficientes..." (p. 94). 
En la gráfica 3 aparecen las participaciones de la nómina total en el valor agregado correspondiente a cada grupo de tamaños. Los puntos $A$ y $B$ muestran las participaciones medias en 1965 y 1970, respectivamente. En todos los tamaños, salvo los menores, las participaciones descienden entre los dos años. Se observa también que la participación má-

Gráfica 3

Participación del Salario en El Valor agregado y tasa DE GANANCIAS POR VALOR DE ACTIVOS POR TRABAJADOR

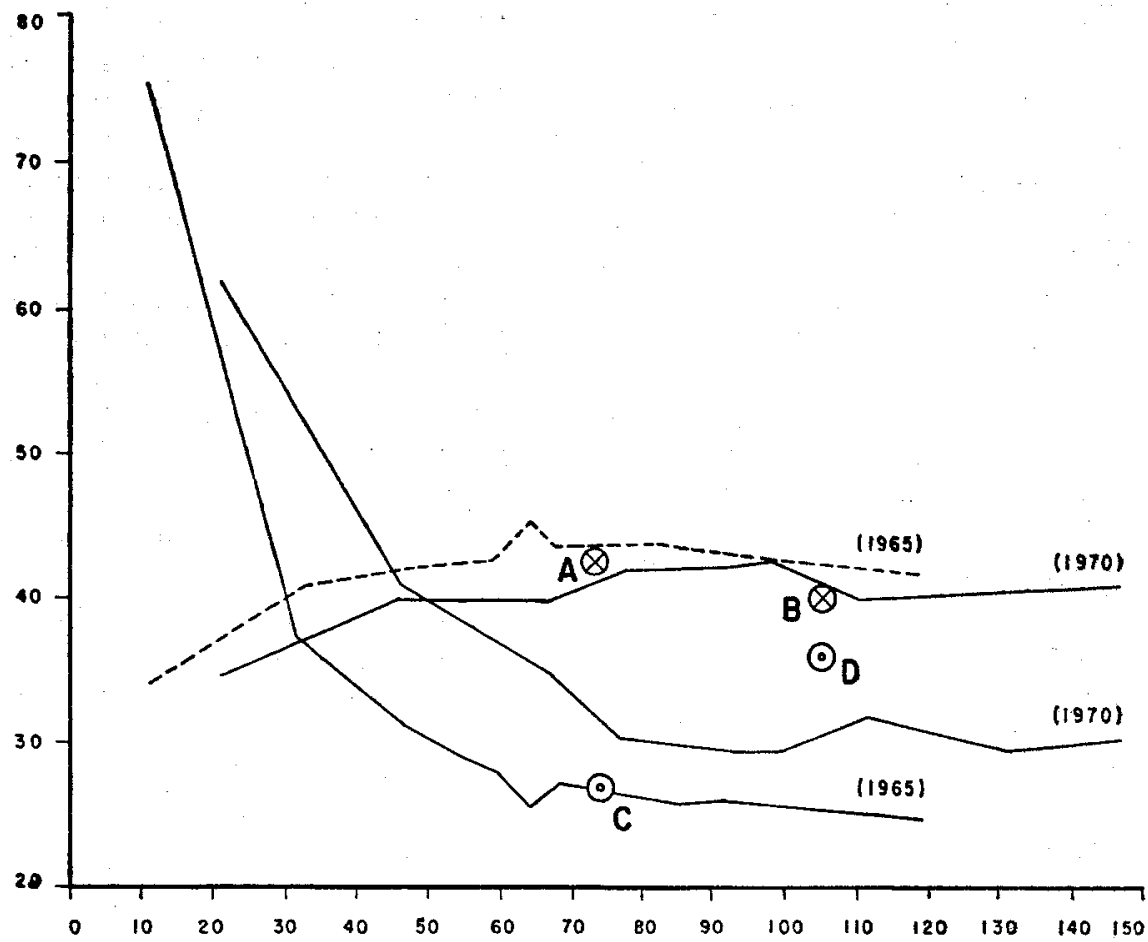

xima ocurre en los tamaños intermedios y la mínima en los más pequeños. ${ }^{9}$ Sin embargo, las diferencias entre los tamaños intermedios y superiores no son muy altas. La gráfica 3 , construida con los datos del cuadro 4, contiene también la relación entre ingresos no salariales (valor agregado menos nómina total) y el monto de los activos por obre-

3 Estos resultados son opuestos a los de Trejo, op. cit., debido en parte a que este último imputa parte del salario a los empresarios y construye un agregado con las empresas menores de 50 obreros. En el trabajo presente se han dejado las cifras tal cual. Tampoco se han analizado niveles de economías de escala por tamaños debido a que en general son indistinguibles del cambio técnico y cualquier otro factor exógeno de cambio en la producción. 
ro. Este último indicador a sido utilizado como aproximación a la tasa de ganancia.

La conducta de las participaciones por tamaños puede deberse a la influencia de cada grupo sobre los precios, a la tecnología de producción, a barreras al crecimiento, al desempleo y al monopolio en el mercado de trabajo, a diferencias regionales, a presiones laborales, $\mathrm{o}$ a discrepancias regionales en los salarios mínimos y su observancia. En las empresas pequeñas en particular es probable que su ubicación en zonas de salarios mínimos bajos y la evasión de la ley explique la menor participación de la nómina en el valor agregado.

El descenso entre los dos años es mayor en las empresas intermedias, es decir, en aquellas donde las participaciones son más altas. En las empresas de 100 a 500 obreros, que representan alrededor de $40 \%$ de la mano de obra, la participación desciende en casi $8 \%$. Por industrias, las participaciones descienden sustancialmente en alimentos, bebidas, muebles, cuero, hule, petróleo y maquinaria no eléctrica, según puede verse en el lado derecho del cuadro 3. Puede verse en este último cuadro que los descensos en la participación llegan hasta $22 \%$ en las industrias de derivados del petróleo.

En la gráfica 3 puede verse que la tasa de ganancia disminuye con el tamaño, primero en forma muy acelerada hasta los tamaños 1-25 y y luego permanece aproximadamente constante. Las tasas de ganancias, calculadas de acuerdo con la definición anterior, muestran un desplazamiento hacia arriba. Salvo por los dos tamaños menores, en todos los restantes la tasa de ganancias aumenta entre los dos años con el nivel de activos por obrero. Esta conducta desde luego corresponde al descenso de las participaciones salariales.

La estructura de participaciones del trabajo y el capital en el valor agregado por tamaños indica que el impacto de un cambio en los salarios sobre los precios es menor que el de un cambio en la tasa de ganancias. Dicho impacto es además decreciente. Por otra parte, la distribución subsecuente de los pagos al capital entre intereses, regalías, dividendos, precios y amortización del equipo, impuestos, etc., constituyen una fuente múltiple de presiones en la tasa de ganancias y de influencias sobre el nivel de tecnología. Al parecer las empresas con mayor participación salarial hacen más por reducir dicha participación manteniendo un crecimiento bajo del empleo y el salario. Este punto será estudiado en mayor detalle en la sección IV.

Si la distribución de pagos a los factores descrita se identifica con grupos de fuerza de trabajo bien definidos, podríamos concluir que la desigualdad del ingreso se origina en la distribución funcional. Sin embargo, para algunos autores el proceso es mucho más complicado, de tal manera que las ganancias por capital no son directamente identificables con ingresos altos, ni las ganancias por trabajo con ingresos bajos. Las cifras muestran en efecto grandes diferencias salariales entre empresas 
de distintos tamaños y otro tanto sucede con gran probabilidad con las ganancias.

Si se hace el supuesto de que las empresas pequeñas tienen un solo empresario y que la propiedad de las grandes es un múltiplo de las pequeñas, se obtienen las cifras de las dos últimas columnas del cuadro 4, que muestran la estructura del volumen de ganancias. Al hacer tal supuesto, en 1965 las empresas grandes tenían ganancias brutas "por empresario" 1157.5 veces mayores a las de las pequeñas, y 1064.9 veces en 1970. Se observa entre los dos años que los múltiplos aumentan hasta el tamaño 251-500 y descienden ligeramente en el último grupo. La interpretación de estos coeficientes es que las empresas grandes necesitarían tener cada una cuando menos 1157.5 propietarios en 1965 y 1064.9 en 1970 para que las ganancias medias por propietario fuesen iguales a las de empresas de tamaño menor. Se observa también que las ganancias de las empresas pequeñas son semejantes a los salarios medios de las más grandes. De aquí podríamos inferior que hay asalariados con ingresos mayores a algunos ingresos por capital. Sin embargo, en todos los casos el salario medio de cada tamaño es menor que las ganancias brutas, lo cual da idea de la magnitud de las transferencias que el mercado de capitales debería realizar para igualar los ingresos (las empresas grandes obtienen ganancias brutas unas 1100 veces mayores al salario medio). Si estas empresas grandes tuviesen un solo propietario éste ganaría un máximo de 1200 veces el ingreso medio de sus obreros.

De acuerdo con estas consideraciones, puede enumerarse el siguiente resumen de características de la industria desde el punto de vista distributivo:

1) aunque se han evitado las comparaciones de cifras absolutas, el crecimiento de las manufacturas mexicanas se ha debido en mayor medida al crecimiento de las industrias existentes, y no a la creación de nuevas empresas (véase el cuadro 1);

2) el crecimiento está vinculado con un notable crecimiento del capital por obrero ( $48 \%$ entre los dos años) que aumenta con el tamaño de la empresa;

3) la estructura de tamaños de empresas determina las disparidades entre los ingresos por capital;

4) la estructura de tamaños de empresas determina las disparidades salariales;

5) la estructura de tamaño determina las disparidades entre ingresos por capital;

6) la norma de crecimiento no es interpretable de acuerdo con los supuestos neoclásicos, a menos que se admita que las empresas mayores tienen índices de eficiencia menor a las menores; ${ }^{10}$

10 A estas observaciones puede añadirse que la relación entre el cambio proporcional de la productividad y el capital por obrero es mayor que uno, contrario a las inferencias de la teoría de la producción. (Véase la columna 6 del cuadro 4.) 
7) el impacto del salario sobre la estructura de costos es menor que el de las ganancias;

8) las tasas brutas de ganancia no son iguales entre empresas grandes y pequeñas aunque sí parecen serlo aproximadamente entre las de tamaño intermedio y mayor. Esto sugiere una explicación de cómo sobreviven las empresas pequeñas, ya que pueden ajustar sus tasas de ganancias dentro de un margen amplio con respecto a las grandes;

9) la participación de los salarios en el valor agregado desciende en el tiempo, y más rápido en las empresas de mayor costo del trabajo.

\section{PARTicipación de los Salarios y Capital POR ObRero}

Hay varias formas de justificar la relación entre el capital por obrero y el salario. Una de ellas es la de la teoría neoclásica, que expresa una relación enteramente física en función de la sustituibilidad técnica entre trabajo y máquinas. Otra justificación surge de suponer que el capital es un vehículo de control de la fuerza de trabajo, por lo cual el salario es establecido al mínimo por la competencia entre los obreros y el desempleo. Puede suponerse también que los obreros asociados a una mayor cantidad de equipo tienen mayor productividad física por razón del mayor uso de energía no humana. Puede también suponerse que al aumentar el capital por obrero, aumenta la responsabilidad de este último en cuanto al uso adecuado de las máquinas y en las funciones del trabajo en general. Algunos enfoques empíricos acuden al principio anterior para analizar diferencias salariales. La discrepancia entre los enfoques se vuelve fundamental cuando se trata de adjudicar a las leyes físicas la determinación del nivel general de salarios y ganancias.

De cualquier manera es posible examinar la relación. entre los cambios en el capital por obrero y los salarios bajo interpretaciones alternativas. La participación del salario en el valor agregado será llamada $a$ y es idéntica a la relación $(a \equiv W L / V \equiv w / p)$ entre el salario $(w)$ y la productividad $(p)$, donde $V$ es el valor agregado y $L$ el número de obreros. Los aumentos en el capital por obrero, si se transmiten directamente en aumentos en la productividad, significan aumentos en los salarios, de acuerdo con la relación $\dot{w} / w=(1-a) \quad(K / K)=(1-w / p) \quad(K / K) .^{11}$ Mientras menor sea la relación salarios/productividad mayor será el efecto de un aumento en el capital por obrero sobre la tasa de crecimiento de los salarios. Esto resulta simplemente del hecho de que la base de los cambios en el salario es menor que la de cambios en la productividad. Sin embargo, para que esta conclusión sea válida es necesario suponer que el empleo de las empresas crece a la misma velocidad que el

11 E1 punto sobre la variable representa el cambio con respecto al tiempo. 
valor agregado, es decir, que la productividad es constante. En caso contrario el cambio en el capital por obrero estará acompañado de sustitución de trabajo por capital. Mientras mayor sea esta sustitución, los salarios aumentarán menos $\mathrm{v}$ el empleo más. De este modo, el salario crecerá a una tasa $\dot{w} / w=A(1-a)(\dot{k} / k)$. Sin embargo, el capital por obrero no es en general la única fuente de cambio del salario. Es posible definir otras causas de cambio asociadas con la importancia de la nómina en el valor agregado. Mientras mayor sea la participación del trabajo en la estructura de costos mayor será la resistencia de los empresarios a alzas en los salarios. Por lo tanto, la relación podría escribirse:

$$
\dot{w} / w=A(1-a) \dot{k} / k+B(1-a)+C
$$

Si en esta ecuación, $A$ se interpreta como la recíproca de la elasticidad de sustitución, la relación corresponde a un teorema neoclásico bajo los supuestos de mercados perfectos y economías de escala nulas. Sin embargo, la relación puede especificarse sin necesidad de acudir a tales supuestos de tal manera que no haya compromiso con una teoría específica de los precios. Se le ha dado la forma anterior solamente para hacer algunas analogías. En el cuadro 5 aparece dicha relación estimada por regresión para cada uno de los 9 tamaños de empresas, tomando las tasas de crecimiento entre 1965 y 1970 de las empresas de cada tamaño observadas a dos dígitos.

Sólo en cuatro casos se encuentra una asociación positiva entre el capital por obrero y el salario, y en todos ellos la variación explicada es muy pequeña. Por otra parte, si el coeficiente $A$ se interpreta como el recíproco de la elasticidad de sustitución, por los valores obtenidos se encuentra que dicha elasticidad tiene un valor muy elevado: de casi 4 en las empresas menores y alrededor de 2 en las restantes. Los aumentos en el capital por obrero se traducen así en pequeños aumentos en el salario. Se encuentra además que los salarios no parecen estar asociados con la importancia de las participaciones. Sin embargo, este procedimiento es todavía muy indirecto.

Para estudiar las hipótesis más en detalle se estimó la relación entre los cambios en las participaciones y el capital por obrero, de acuerdo con los mismos principios aue la relación anterior:

$$
\dot{a} / a=A(1-a) \dot{k} / k+B(1-a)+C
$$

Los resultados aparecen en el cuadro 6 y muestran ahora una relación nula con el capital por obrero. Sin embargo, esta vez aparecen más regresiones significativas y en todos los casos $B$ y $C$ son los coeficientes significativos. Estos resultados sugieren que las empresas reducen la participación de los salarios en el valor agregado mientras mayor es dicha 
Cuadro 5

COEFICIENTES DE REgRESIÓN SALARIOS-ACTIVOS *

\begin{tabular}{|c|c|c|c|c|c|}
\hline Tamaño ${ }^{b}$ & $A$ & $B$ & $C$ & $R^{2}$ & $F$ \\
\hline $1-5$ & $\begin{array}{l}0.2735^{\circ} \\
(4.28)\end{array}$ & $\begin{array}{l}0.4814 \\
(0.70)\end{array}$ & $\begin{array}{l}0.0722 \\
(0.15)\end{array}$ & 0.55 & $9.65^{\mathrm{e}}$ \\
\hline $6-15$ & $\begin{array}{l}0.2009 \\
(1.00)\end{array}$ & $\begin{array}{l}0.8401 \\
(0.94)\end{array}$ & $\begin{array}{l}-0.1209 \\
(-0.07)\end{array}$ & 0.07 & 0.65 \\
\hline $16-25$ & $\begin{array}{l}0.2990 \\
(1.66)\end{array}$ & $\begin{array}{l}-0.0994 \\
(-0.15)\end{array}$ & $\begin{array}{l}0.4545 \\
(1.18)\end{array}$ & 0.16 & 1.50 \\
\hline $26-50$ & $\begin{array}{l}0.5311^{\mathrm{c}} \\
(3.19)\end{array}$ & $\begin{array}{l}0.4878 \\
(1.64)\end{array}$ & $\begin{array}{l}0.0066 \\
(0.04)\end{array}$ & 0.41 & $5.17^{e}$ \\
\hline $51-75$ & $\begin{array}{l}0.1888 \\
(1.80)\end{array}$ & $\begin{array}{l}-0.7896 \\
(-1.56)\end{array}$ & $\begin{array}{l}0.7696 \\
(2.76)\end{array}$ & 0.27 & 2.90 \\
\hline $76-100$ & $\begin{array}{l}0.0621 \\
(0.13)\end{array}$ & $\begin{array}{l}0.2171 \\
(0.28)\end{array}$ & $\begin{array}{l}0.3740 \\
(0.80)\end{array}$ & 0.01 & 0.04 \\
\hline $101-250$ & $\begin{array}{l}0.5532^{\mathrm{c}} \\
(2.70)\end{array}$ & $\begin{array}{l}0.4393 \\
(0.86)\end{array}$ & $\begin{array}{l}0.0766 \\
(0.26)\end{array}$ & 0.32 & $3.82^{\mathrm{c}}$ \\
\hline $251-500$ & $\begin{array}{l}0.0674 \\
(0.44)\end{array}$ & $\begin{array}{l}0.3033 \\
(0.85)\end{array}$ & $\begin{array}{l}0.3132 \\
(1.14)\end{array}$ & 0.03 & 0.24 \\
\hline Más de 500 & $\begin{array}{l}0.4756^{\mathrm{c}} \\
(2.48)\end{array}$ & $\begin{array}{l}0.6987 \\
(1.29)\end{array}$ & $\begin{array}{c}0.0369 \\
(-0.11)\end{array}$ & 0.32 & $3.10^{\mathrm{c}}$ \\
\hline Total de empresas & 0.4474 & 0.3032 & 0.5695 & 0.22 & 2.19 \\
\hline
\end{tabular}

a $\dot{w} / w=A(1-a) \dot{k} / k+B(1-a)+C$. Los números entre paréntesis son los coeficientes $t$ de student.

- Tamaño de los establecimientos según el número de trabajadores.

c Coeficientes de regresión significativos al $2.5 \%$.

participación. ${ }^{12}$ Lo mismo se observa en los términos constantes que son todos negativos. En la terminología neoclásica estos cálculos mostrarían que el cambio técnico es "sesgado" en el sentido de ahorrar trabajo. Sin embargo, este tipo de interpretación parte del supuesto de que la tecnología es exógena y de que los factores son remunerados al monto de sus productividades marginales en mercados perfectos. Para interpretar la relación o para especificarla no es necesario acudir a una teoría de la determinación del salario. Las cifras muestran simplemente que las empresas buscan reducir los costos del trabajo mientras mayores sean éstos, pero no permiten inferir cuál es su causa.

12 Nótese que $B$ es el coeficiente de (1-a), de tal manera que los aumentos en $a$ reducen el salario a la tasa $\dot{a} / \mathbf{a}$. Esta especificación corresponde con la del modelo neoclásico con cambio técnico autónomo no neutro. Véase Bliss, op. cit., Cap. 9. 
Cuadro 6

COEFICIENTES DE REGRESIÓN ENTRE PARTICIPACIONES DE LA NÓMINA EN EL VALOR AGREGADO Y ACTIVOS POR OBRERO ${ }^{\text {a }}$

\begin{tabular}{|c|c|c|c|c|c|}
\hline Tamaños & $A$ & $B$ & $C$ & $R^{2}$ & $F$ \\
\hline $1-5$ & $\begin{array}{l}0.0165 \\
(0.33)\end{array}$ & $\begin{array}{l}1.8079^{b} \\
(3.37)\end{array}$ & $\begin{array}{l}-1.1680^{b} \\
(-3.14)\end{array}$ & 0.44 & $6.2^{b}$ \\
\hline $6-15$ & $\begin{array}{l}-1.1026 \\
(-1.53)\end{array}$ & $\begin{array}{l}0.8775^{b} \\
(2.94)\end{array}$ & $\begin{array}{l}-0.4790^{\mathrm{b}} \\
(-2.61)\end{array}$ & 0.55 & $9.7^{b}$ \\
\hline $16-25$ & $\begin{array}{l}-0.3267 \\
(-2.36)\end{array}$ & $\begin{array}{c}0.1370 \\
(0.27)\end{array}$ & $\begin{array}{l}-0.0016 \\
(-0.01)\end{array}$ & 0.28 & $3.1^{\mathrm{b}}$ \\
\hline $26-50$ & $\begin{array}{l}0.0550 \\
(0.20)\end{array}$ & $\begin{array}{l}1.3657^{b} \\
(2.75)\end{array}$ & $\begin{array}{c}-0.7649^{b} \\
(12.58)\end{array}$ & 0.36 & $4.3^{\mathrm{b}}$ \\
\hline $51-75$ & $\begin{array}{l}0.1282 \\
(0.87)\end{array}$ & $\begin{array}{r}0.2725 \\
(0.38)\end{array}$ & $\begin{array}{l}-0.2219 \\
(-0.56)\end{array}$ & 0.05 & 0.4 \\
\hline $76-100$ & $\begin{array}{l}-0.1924 \\
(-0.60)\end{array}$ & $\begin{array}{l}1.0857^{\mathrm{b}} \\
(2.03)\end{array}$ & $\begin{array}{l}-0.5348^{b} \\
(-1.66)\end{array}$ & 0.29 & $3.0^{\mathrm{b}}$ \\
\hline $101-250$ & $\begin{array}{l}-0.0750 \\
(-0.41)\end{array}$ & $\begin{array}{l}0.3572 \\
(0.77)\end{array}$ & $\begin{array}{l}-0.2304 \\
(-0.88)\end{array}$ & 0.05 & 0.4 \\
\hline $251-500$ & $\begin{array}{l}-0.0715 \\
(-0.42)\end{array}$ & $\begin{array}{l}0.8560 \\
(1.65)\end{array}$ & $\begin{array}{l}-0.4987 \\
(-1.64)\end{array}$ & 0.19 & 1.9 \\
\hline Más de 500 & $\begin{array}{l}0.0423 \\
(0.40)\end{array}$ & $\begin{array}{l}0.7974^{\mathrm{b}} \\
(2.70)\end{array}$ & $\begin{array}{l}-0.4686^{b} \\
(-2.59)\end{array}$ & 0.41 & $4.6^{b}$ \\
\hline $\begin{array}{l}\text { Total de } \\
\text { establecimientos }\end{array}$ & $\begin{array}{l}0.1285 \\
(1.04)\end{array}$ & $\begin{array}{l}-0.0399 \\
(-0.11)\end{array}$ & $\begin{array}{l}-0.0486 \\
(-0.23)\end{array}$ & 0.07 & 0.6 \\
\hline
\end{tabular}

a $\dot{a} / a=A(1-a) \dot{k} / k-B(1-a)+C$. Los números entre paréntesis son los coeficientes $t$ de student.

b Coeficientes significativos al $2.5 \%$.

\section{RESUMEN Y CONCLUSIONES}

Las cifras y cálculos examinados muestran varias direcciones posibles de la investigación sobre distribución del ingreso en las fuentes primarias. Deliberadamente se ha evitado proponer pruebas de hipótesis de teorías específicas, de tal manera de dar a los resultados un carácter enteramente descriptivo.

El crecimiento industrial de México, tan citado como logro y defecto de la política de crecimiento, ciertamente es origen de cambios en la distribución del ingreso. Es un hecho que la política de industrialización ha desatendido el fenómeno de concentración tanto en el sentido de los 
tamaños como en el geográfico. Los elevados niveles de concentración industrial se sugieren como causa de la desigualdad entre e intra salarios y ganancias, que conduce a una mayor concentración del ingreso personal. Se encuentra que la concentración industrial está asociada con disparidades del ingreso en todas direcciones. Los resultados sugieren específicamente que mediante la reducción de la desigualdad de tamaños de la industria es posible lograr cambios sustanciales en la distribución funcional del ingreso en favor del trabajo, y desde luego reducir diferencias salariales y de ingresos por capital. Esta conclusión es equivalente a la de que una reasignación de las inversiones puede conducir a una reducción de la desigualdad del ingreso personal.

Las gráficas de productividad y salarios muestran escasa coincidencia con los supuestos neoclásicos de curvatura de las funciones de producción. En el caso de las participaciones salariales el poder explicativo de las regresiones estimadas, aunque bajo, es atribuible a "otros factores" y no a la relación capital/trabajo. Esta conclusión, de que son otros factores los que explican cambios en la distribución funcional, equivale a inferir que el análisis de productividades marginales tiene poco valor explicativo. Por otra parte, la relación entre el cambio en las participaciones salariales y el nivel de éstas sugiere el camino hacia investigaciones más específicas sobre la determinación del salario y el nivel de empleo. Cabe señalar que el nombre "cambio técnico" o "eficiencia" que suele darse a los otros factores no tiene mayor significado. Solamente se refiere a cambios en la estructura de producción disociados de cambios en el monto de los insumos.

Puede parecer que los ejercicios estadísticos presentados no pueden hacer justicia a la prueba empírica de teoría alguna. Esta apreciación es correcta. Sin embargo, las cifras sugieren qué puede esperarse de la aplicación de métodos estadísticos más refinados. Las conjeturas sobre si la tasa de ganancias coincide o no con el valor de la productividad marginal del capital no pueden ir muy lejos. Llevar el análisis al estudio microeconómico de la empresa puede resultar muy productivo para entender la injerencia de la producción y otras cuestiones de administración de empresas. Sin embargo, es improbable que ayude en algo a explicar la determinación del salario y las distribuciones funcional o personal.

Por último, conviene señalar de nuevo el error de inferir de la no coincidencia de valores y productividades, que lo que debe hacerse es eliminar imperfecciones en los mercados, como el socorrido caso de las uniones sindicales y sus efectos sobre el salario y el empleo. Este tipo de inferencias no sólo son valorativas, sino que parten del hecho de que la teoría no explica los fenómenos. 